\title{
Axle Load Distribution Characterization for Mechanistic Pavement Design
}

\author{
S.A.S.L. Sumanasekara and W.K. Mampearachchi
}

\begin{abstract}
In Sri Lanka, Overseas Road Note 31 and AASHTO method are the most frequently used pavement design methods. However, in these methods the consideration given for utilization of readily available in-situ materials and incorporating characteristics of substandard materials into the designs, is not significant. On contrary, the AASHTO Mechanistic-Empirical (M-E) method of pavement design allows the designer to utilize in-situ materials and allows to optimize the pavement design to suit the conditions at site. However, due to the amount of data input required in M-E design, the preference of local practitioners in using the M-E method is not satisfactory. This study was carried out with the objective of identifying trends in axle load group type distributions of different regions in Sri Lanka. It has been found that there are similar patterns in distribution of axle group types throughout the island. Also, it was found that there are certain geographical regions with similarities in pavement loading conditions. An attempt for zoning traffic load distributions for selected geographic areas is presented in this study. This will aid in determining the traffic data input for M-E design, with a degree of accuracy level 2 as defined in M-E design method.
\end{abstract}

Keywords: Axle load distribution, Traffic load zoning, Mechanistic-Empirical pavement design

\section{Introduction}

As per the World Bank records of year 2018, Sri Lanka had the highest road density among the South Asian countries with $173.9 \mathrm{~km}$ of roads per 100 square kilometres of land. Sri Lankan government reserves a considerable amount of finance every year for developing and maintaining the existing road infrastructure, which is a well-recognized national priority. In the currently used methods for local pavement designs, utilization of the readily available insitu materials is not widely seen. Also, consideration given to incorporate substandard materials is not prominent. Therefore, in most of the occasions, the economics of the construction processes have been adversely affected. The AASHTO M-E method of pavement design combines the physical causes such as stresses, strains, and deflections within a pavement structure and the empirical mathematical models. The pavement responses under M-E method are computed using detailed traffic loading, material properties, and environmental data, and are used to predict the incremental damage over time. The design of pavements under M-E method is an iterative process using analysis results based on trial designs postulated by the designer. Pavement design using M-E approach has been well developed since the release of Mechanisticempirical Pavement Design Guide (MEPDG) in 2002 [1]. The basic advantage of combined M-E method over a purely Empirical method is the ability to accurately characterize in situ material. Hence, it provides practical designs for the given conditions. M-E method can be used in designing new pavements and also in designing overlays for existing pavements. It has been proven through previous studies that M-E approach in designing pavements provides more reliable performance predictions with more realistic and optimized designs than the empirical methods $[2,3]$.

\section{Literature Review}

\subsection{Mechanistic-Empirical Pavement Design}

A mechanistic approach refers to physical causes to explain phenomena such as stresses, strains, and deflections within a pavement structure. The physical causes in a pavement are the load and material properties of the structure. Traffic loading is a heterogeneous mix of vehicles, axle types, and axle loads, with distributions that vary with time throughout the day and over the pavement design life. Pavement materials respond to these loads in complex ways influenced by stress state and magnitude, temperature, moisture, time, loading rate, and other factors [4]. Mathematical models had been used to describe the relationship between these phenomena and their physical causes.

Eng. S.A.S.L. Sumanasekara, B.Sc. Eng. (Hons) (Moratuwa),MBA(UK),AMIE(SL), Project Engineer, Road Development Authority

E-mail:shanikasumanasekara347@gmail.com

Eng. (Prof.) W.K. Mampearachchi, B.Sc. Eng., MSCE (S. Florida),Ph.D.(Florida), CEng, MIE(SL), Professor in Civil Engineering, University of Moratuwa

e-mail:wasanthak@uom.lk 
Along with the mechanistic approach, empirical elements are used when defining what value of the calculated stresses, strains and deflections result in pavement failure. The relationship between physical phenomena and pavement failure is described empirically by derived equations that compute the number of loading cycles to failure.

\subsection{Importance of Traffic Loads on Pavement Design}

The pavement design reliability is defined as "the probability that the pavement's traffic load capacity exceeds the cumulative traffic loading on the pavement during a selected design life" [5].

Traffic axle loads and volumes are very crucial parameters in the design of pavements. In a study done to assess the impact of reliability of inputs on the M-E design has shown that the axle weight variability has an overwhelming effect on the variability of either fatigue or rutting performance predictions. The study has used Monte Carlo simulation and the investigation has been done on a typical threelayer pavement cross section consisting of Asphalt Concrete (AC) over granular base on top of subgrade soil. Traffic load distributions, obtained from Weigh-in-Motion (WIM) sites had been utilized for the study and it had been found that when the loads applied are constant, the variability of predicted fatigue performance depends on the thickness and the stiffness of the AC layer. Further, the study states base thickness and the subgrade modulus have a minor effect on the output variability. This is acceptable as the fatigue transfer function for M-E design is based on tensile strain at the base of the AC layer. Therefore, in M-E design, the AC parameters take precedence over the parameters of base and subgrade. They have also found that though the pavement structural parameters contribute to output variability, the traffic weight is the single most important input parameter [6].

The M-E pavement design requires to assume axle load spectrum data. It allows three levels of hierarchical approaches to assign traffic inputs for the design procedure [1].

Level 1: Highest accuracy in data with sitespecific axle load and traffic volume data collected at or near the project

Level 2: Intermediate accuracy in data, with average knowledge of traffic characteristics with regional axle load data and site-specific volume data
Level 3: Least accuracy. Use knowledge of state-wide default vehicle weight and volume data.

Gathering level 1 traffic data is straightforward and requires a lot of resources. In general, traffic volume is easier to obtain than load spectrum considering the time and resource consumption, in gathering data. On many existing routes, the designers are more likely to have only historical traffic counts and vehicle classifications. For a new route, historical traffic data may not exist at all. Under these circumstances, it is necessary to estimate load spectra based on indirect information. If available, the WIM data in proximity, could be used with some level of confidence for the M-E design process.

Therefore, there is a requirement to identify the traffic load distribution patterns in order to derive representative axle load spectra that can be used with some level of confidence in the ME design process.

\section{Objectives}

The objectives of this study are to determine the trends in axle load distributions of different axle group son pavements and to identify similarities in axle load distributions in different axle load survey locations, to be used in mechanistic pavement designs.

\section{Methodology}

\subsection{Data Collection}

The data include the axle load surveys and manually classified vehicle counts for different roads collected from Road Development Authority and from expressway projects.

\subsection{Method of Analysis}

In order to identify the traffic trends in a region, the Axle type distributions were analysed in the data samples gathered. For this, the axle counts measured at axle load survey stations were classified to their axle group type as follows.

- Single axle/ single tires (SAST)

- Single axle/ dual tires (SADT)

- Tandem axle/ dual tires (TADT)

- Tri axle/ dual tires (TRDT)

The above axle group types were defined as per the Austroad guidelines [7]. The axle counts were then converted to percentages to identify trends in the axle group type distributions.

Clustering techniques have been utilized to identify groups with similarities in pavement loading conditions. Cluster analysis helps in constructing groups (or clusters) while 
ensuring the property that, within a group, the observations are as similar as possible, and the observations belonging to different groups are different as possible [8].

Among the two main clustering techniques, i.e. K-means clustering and Hierarchical clustering, Hierarchical clustering technique was used for this research as the number of clusters were unknown and it is aimed at determining optimal number of clusters (K-Means can be used only when the number of clusters is predefined).

Clustering algorithms use the distance in order to separate observations into different groups. Here, Euclidian Distance, $e$ was used. Euclidian Distance is defined as the distance between attributes for each pair of objects. If two attributes $I\left(x^{1}, y^{1}\right)$ and $j\left(x^{2}, y^{2}\right)$ are plotted in a Cartesian coordinate plane, the Euclidian distance $e_{i j}$ would be linear distance between two attributes defined on the plot by their coordinates.

$e_{i j}=\sqrt{\left(x_{1}-x_{2}\right)^{2}+\left(y_{1}-y_{2}\right)^{2}}$

A value of $e_{i j}$ closer to 0 suggests that there is a similarity between the pair of objects/ attributes.

A clustering tree is to be constructed so that pairs of objects will be sequentially grouped together (This is called Ward's Minimum Variance method). The steps in hierarchical clustering are listed as follows.

Step 1:

Start by including every point into its own cluster. The axle load spectra for all the axle load survey locations were treated as clusters. The axle loads were set into axle load bins with a range of $5 \mathrm{kN}$. Axle count in the load bins were converted to percentages so that all the values compared will be standardized as required by hierarchical clustering.

Step 2:

Then the two points that are closest to each other based on the distances from the distance matrix were merged together and an average is obtained. The consequence would be that there is one less cluster.

Step 3:

Then the distances between the new (i.e. the averaged cluster) and old clusters were recalculated and saved in a new distance matrix which will be used in the next step.

Step 4:

Steps 1 and 2 were repeated until all clusters were merged into a reduced number of clusters, each containing all suitable, related points. Consideration was given to form a number of clusters which were similar and could be utilized to define a region with a similar axle load distribution.

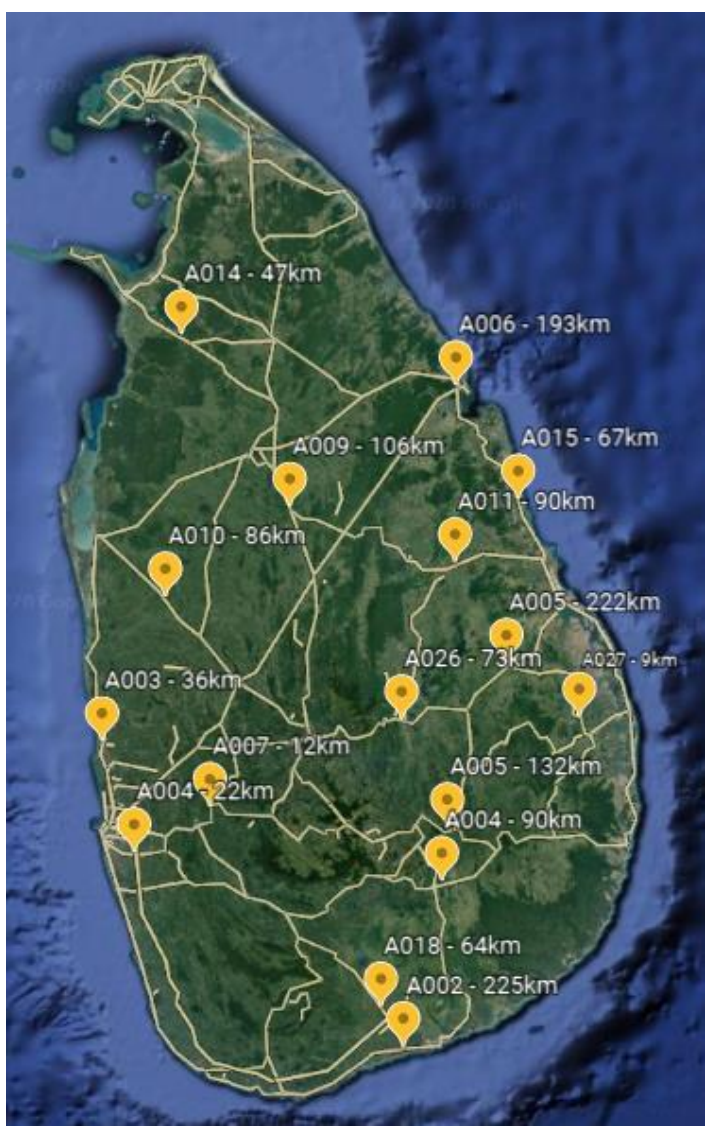

Figure 1- Axle Load Survey Locations

For this cluster analysis, the 'IBM SPSS Statistics 25' software [9] was used.

\section{Analysis}

\subsection{Axle Load Survey Locations}

The axle load survey data from the 16 axle load survey locations shown in Figure 1 were collected from Road Development Authority and highway projects.

\subsection{Analysis of Trends in Axle Loads on Pavements}

\subsubsection{Distribution of Axle Counts}

A summary of the axle counts under each axle group types at the surveyed locations is presented in Table 1 . The axle counts under each axle group type were converted into percentages to make the data more rational in analysing. Accordingly, Table 2 presents the percentages of axle counts under each axle load group.

From Table 2, it can be seen that about $57 \%$ of the axles were single axles with single tire. About 38\% were single axles with dual tires. The tandem axles with dual tires count to about $5 \%$ and the tri axles with dual tires were $0.1 \%$. It can be seen that the contribution of tri axle 
dual tires is negligible and TRDT was not considered in further analysis of this research.

Table 1 - Axle Counts under Each Axle Group Types

\begin{tabular}{|c|c|c|c|c|c|}
\hline \multirow{2}{*}{$\begin{array}{c}\text { Axle load survey } \\
\text { location }\end{array}$} & \multicolumn{3}{|c|}{ No. of Axles in Axle group types } & \multirow{2}{*}{$\begin{array}{c}\text { Total no. of } \\
\text { axles }\end{array}$} \\
\cline { 2 - 5 } & SAST & SADT & TADT & TRDT & 900 \\
\hline A002-225km & 538 & 301 & 61 & 0 & 2087 \\
\hline A003-36km & 1102 & 768 & 216 & 1 & 1537 \\
\hline A004-22km & 928 & 550 & 57 & 2 & 1360 \\
\hline A004-90km & 727 & 547 & 84 & 2 & 517 \\
\hline A005-132km & 333 & 181 & 3 & 0 & 500 \\
\hline A005-222km & 297 & 183 & 18 & 2 & 804 \\
\hline A006-193km & 429 & 263 & 111 & 1 & 1351 \\
\hline A007-12km & 790 & 533 & 28 & 0 & 536 \\
\hline A009-106km & 282 & 227 & 27 & 0 & 592 \\
\hline A010-84km & 355 & 219 & 18 & 0 & 876 \\
\hline A011-90km & 476 & 354 & 44 & 2 & 473 \\
\hline A014-47km & 253 & 208 & 12 & 0 & 338 \\
\hline A015-67km & 229 & 97 & 12 & 0 & 644 \\
\hline A018-64km & 349 & 258 & 34 & 3 & 354 \\
\hline A026-73km & 185 & 164 & 5 & 0 & 461 \\
\hline A027-9km & 279 & 154 & 28 & 0 & \\
\hline
\end{tabular}

Table 2 - Percentages of Axle Counts under Each Axle Load Group

\begin{tabular}{|c|c|c|c|c|}
\hline \multirow{2}{*}{ Axle load survey location } & \multicolumn{4}{|c|}{$\%$ of Axles in Axle group types } \\
\hline & SAST & SADT & TADT & TRDT \\
\hline $\mathrm{A} 002-225 \mathrm{~km}$ & 59.78 & 33.44 & 6.78 & 0.00 \\
\hline A003-36km & 52.80 & 36.80 & 10.35 & 0.05 \\
\hline A004 -22km & 60.38 & 35.78 & 3.71 & 0.13 \\
\hline A004 -90km & 53.46 & 40.22 & 6.18 & 0.15 \\
\hline A005-132km & 64.41 & 35.01 & 0.58 & 0.00 \\
\hline A005-222km & 59.40 & 36.60 & 3.60 & 0.40 \\
\hline A006-193km & 53.36 & 32.71 & 13.81 & 0.12 \\
\hline $\mathrm{A} 007-12 \mathrm{~km}$ & 58.48 & 39.45 & 2.07 & 0.00 \\
\hline A009-106km & 52.61 & 42.35 & 5.04 & 0.00 \\
\hline A010-84km & 59.97 & 36.99 & 3.04 & 0.00 \\
\hline A011-90km & 54.34 & 40.41 & 5.02 & 0.23 \\
\hline A014-47km & 53.49 & 43.97 & 2.54 & 0.00 \\
\hline A015-67km & 67.75 & 28.70 & 3.55 & 0.00 \\
\hline $\mathrm{A} 018-64 \mathrm{~km}$ & 54.19 & 40.06 & 5.28 & 0.47 \\
\hline A026-73km & 52.26 & 46.33 & 1.41 & 0.00 \\
\hline $\mathrm{A} 027-9 \mathrm{~km}$ & 60.52 & 33.41 & 6.07 & 0.00 \\
\hline Average & 57.32 & 37.64 & 4.94 & 0.10 \\
\hline Standard deviation & 4.69 & 4.57 & 3.35 & 0.15 \\
\hline
\end{tabular}

\subsubsection{Distribution of Axle Loads}

The graphs of average relative frequency versus the axle load show the shapes and distributions of axle loads. Following presents a discussion on the SAST, SADT, and TADT axle load distributions.

\section{Single Axle/Single Tire}

The relative frequencies of single axle count versus the axle loads in the 16 locations are shown in Figure 2. There are four distinct peaks that can be identified in the graphs. The First two of the peaks occur in between the axle load range $10-18 \mathrm{kN}$. The third peak occurs around $38 \mathrm{kN}$ and the fourth peak occurs around 62 $\mathrm{kN}$. These peaks can be considered to represent the loaded and unloaded conditions of the vehicles. The peak in 10-18 $\mathrm{kN}$ range represents the unloaded situations and the $38 \mathrm{kN}$ and 62 $\mathrm{kN}$ represent the loaded situations. It is also seen that the frequency of the first two peaks is higher than the frequency of occurrence of the second two peaks. The maximum axle load for single axle-single tire is approximately $90 \mathrm{kN}$. 


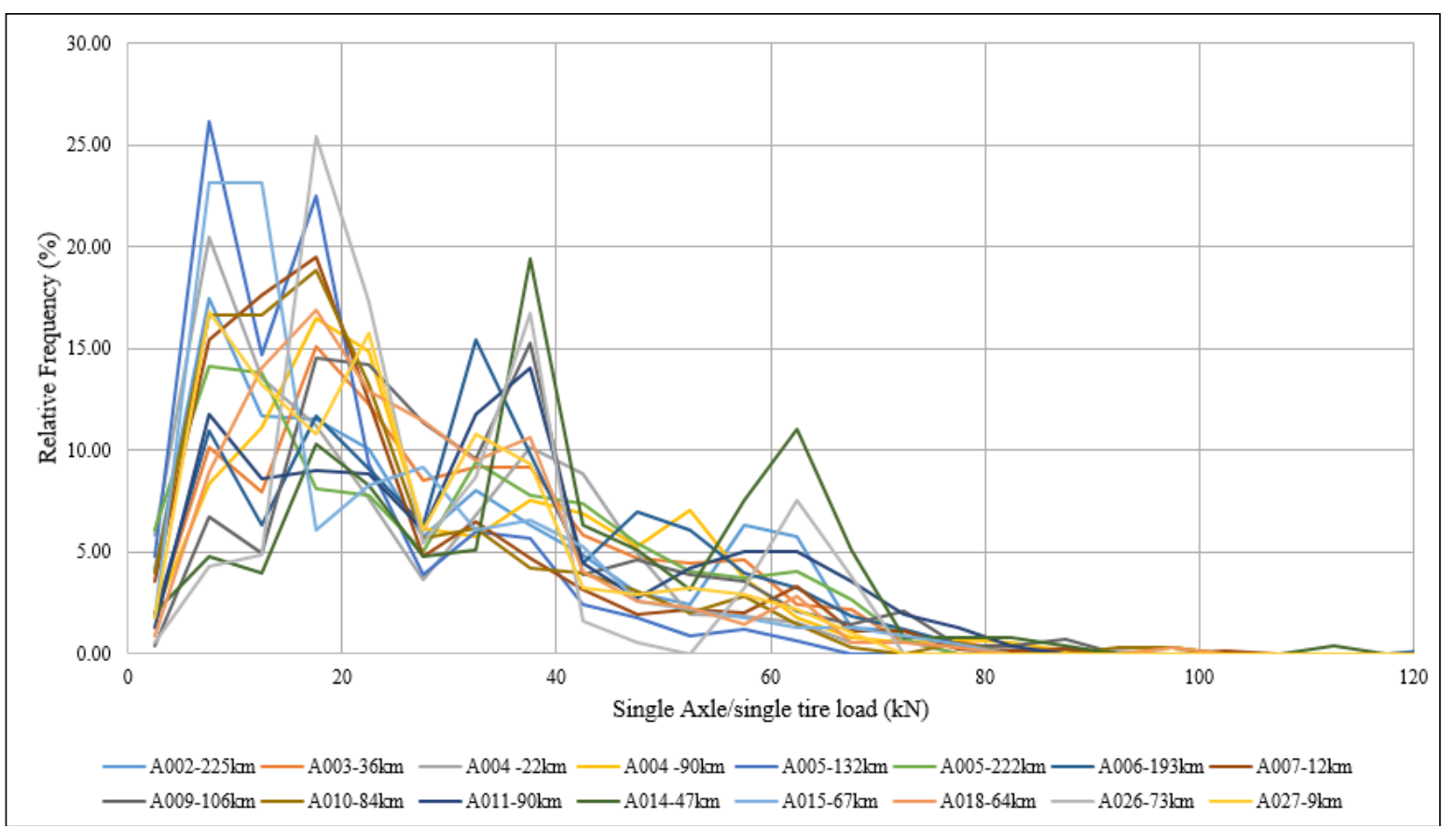

Figure 2 - Relative Frequency vs Single Axle/Single Tire Load

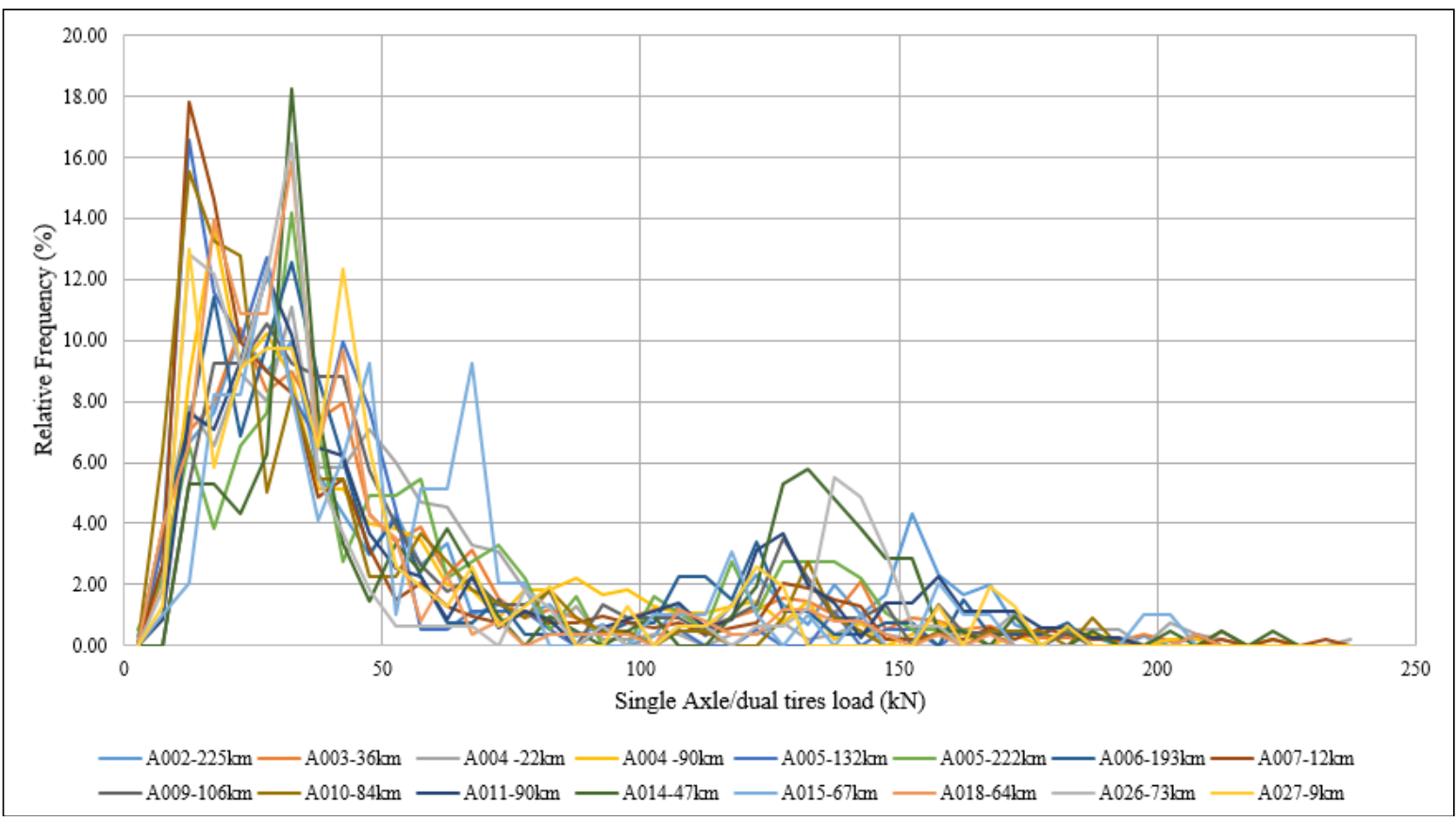

Figure 3 - Relative frequency vs Single Axle/Dual Tires Load

\section{Single Axle/Dual Tires}

The graphs of relative frequency of single axle/dual tires counts versus the axle load are shown in the Figure 3. The Maximum axle load for single axle/dual tire is approximately $200 \mathrm{kN}$. Three peaks can be identified in these graphs. The first two peaks occur in the load range of $15-25 \mathrm{kN}$ and the third peak occurs around $135 \mathrm{kN}$. These peaks can be considered to represent the mean axle loads of a loaded (third peak) and unloaded (first and second peaks) vehicle with single axles having dual tires. It is also seen that the occurrence of the third peak is less frequent than the first and second peaks.

\section{Tandem Axle/Dual Tires}

The graphs showing the relative frequency of tandem axle/dual tire counts versus the axle load are in Figure 4. From these graphs it can be identified that there is a clear peak arising around $60 \mathrm{kN}$ load. However, unlike in the previous plots, it is difficult to identify any other distinct peak in these graphs. 


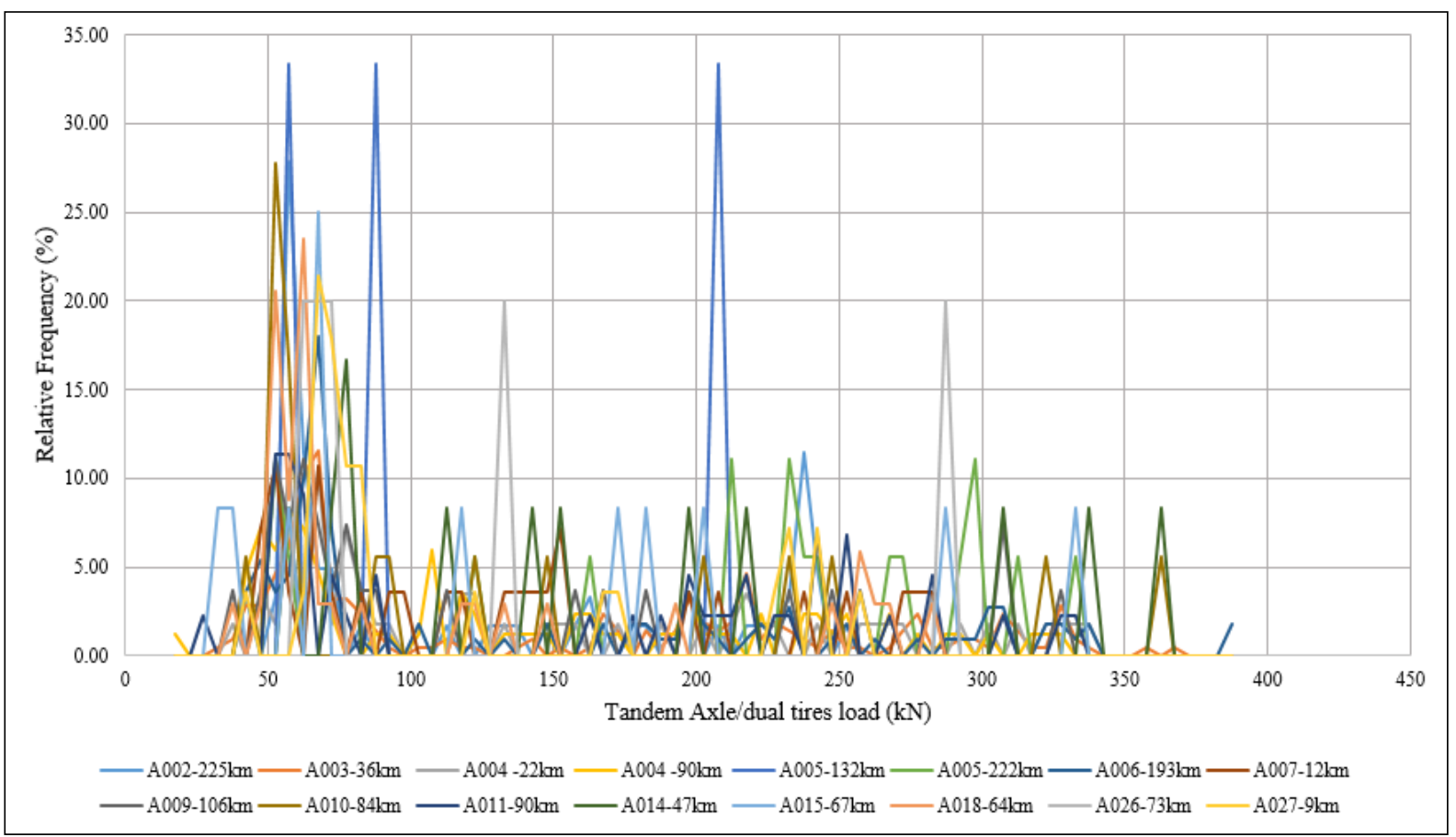

Figure 4 - Relative Frequency vs Tandem Axle/Dual Tires Load

\subsection{Classification of Pavement Loading} Patterns in Regions

For the 16 axle load surveys, a cluster analysis was carried out using IBM SPSS Statistics 25 software. The objects for cluster analysis were the 16 axle load survey locations and the attributes were the values in the load bins.
Following presents the discussion on the cluster analysis.

\section{Single Axle/Single Tire}

The Euclidean matrix for the distributions of SAST loads is in the following Table 3.

Table 3 - Squared Euclidean Distance Matrix for SAST Load Distributions

\begin{tabular}{|c|c|c|c|c|c|c|c|c|c|c|c|c|c|c|c|}
\hline \multirow[b]{2}{*}{ Case } & \multicolumn{15}{|c|}{ Squared Euclidean Distance } \\
\hline & 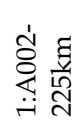 & 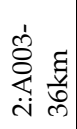 & 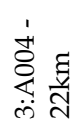 & 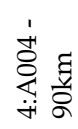 & 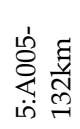 & 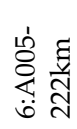 & 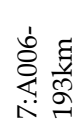 & 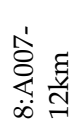 & 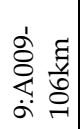 & 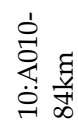 & 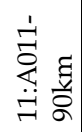 & 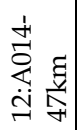 & 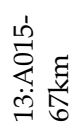 & 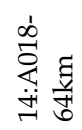 & 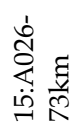 \\
\hline 1:A002-225km & & & & & & & & & & & & & & & \\
\hline 2:A003-36km & 0.013 & & & & & & & & & & & & & & \\
\hline 3:A004 -22km & 0.010 & 0.025 & & & & & & & & & & & & & \\
\hline 4:A004 -90km & 0.020 & 0.005 & 0.030 & & & & & & & & & & & & \\
\hline 5:A005-132km & 0.028 & 0.047 & 0.024 & 0.049 & & & & & & & & & & & \\
\hline $6: \mathrm{A} 005-222 \mathrm{~km}$ & 0.007 & 0.016 & 0.009 & 0.021 & 0.045 & & & & & & & & & & \\
\hline 7:A006-193km & 0.019 & 0.008 & 0.029 & 0.020 & 0.061 & 0.016 & & & & & & & & & \\
\hline 8:A007-12km & 0.014 & 0.021 & 0.021 & 0.017 & 0.016 & 0.023 & 0.038 & & & & & & & & \\
\hline 9:A009-106km & 0.034 & 0.008 & 0.047 & 0.017 & 0.077 & 0.036 & 0.016 & 0.045 & & & & & & & \\
\hline 10:A010-84km & 0.013 & 0.020 & 0.019 & 0.016 & 0.014 & 0.022 & 0.037 & 0.001 & 0.044 & & & & & & \\
\hline 11:A011-90km & 0.015 & 0.011 & 0.024 & 0.023 & 0.061 & 0.013 & 0.008 & 0.037 & 0.015 & 0.038 & & & & & \\
\hline 12:A014-47km & 0.047 & 0.031 & 0.060 & 0.042 & 0.113 & 0.044 & 0.035 & 0.075 & 0.026 & 0.079 & 0.021 & & & & \\
\hline 13:A015-67km & 0.026 & 0.053 & 0.020 & 0.056 & 0.040 & 0.025 & 0.061 & 0.032 & 0.081 & 0.030 & 0.048 & 0.106 & & & \\
\hline 14:A018-64km & 0.021 & 0.008 & 0.032 & 0.011 & 0.046 & 0.024 & 0.021 & 0.016 & 0.013 & 0.017 & 0.020 & 0.047 & 0.046 & & \\
\hline 15:A026-73km & 0.063 & 0.033 & 0.084 & 0.038 & 0.086 & 0.077 & 0.051 & 0.054 & 0.025 & 0.059 & 0.048 & 0.042 & 0.134 & 0.032 & \\
\hline 16:A027-9km & 0.009 & 0.013 & 0.016 & 0.017 & 0.033 & 0.014 & 0.018 & 0.016 & 0.026 & 0.014 & 0.015 & 0.056 & 0.026 & 0.015 & 0.056 \\
\hline
\end{tabular}


In the above Euclidean matrix, the values that are closest to 0.0 are $0.001,0.005$, and 0.007 (values $\leq 0.005$ are considered). Therefore, it can be said that the SAST load distributions of two

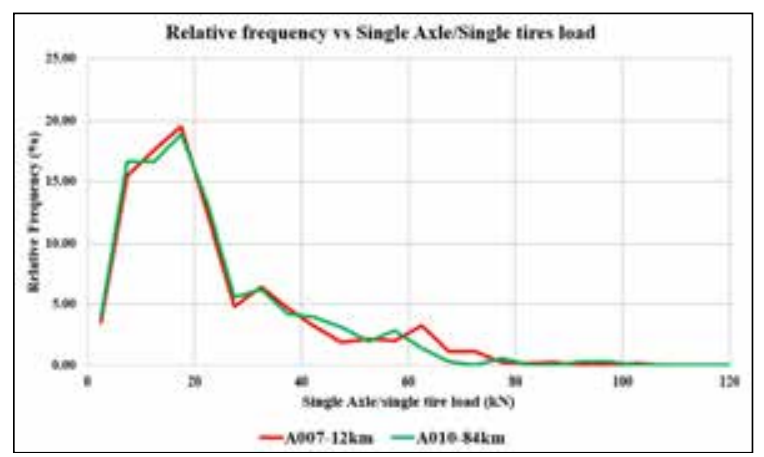

Figure 5 - SAST Axle Load Distribution at Locations A010-84 km and A007-12 km

\section{Single Axle/Dual Tires}

Table 4 presents the Euclidean matrix for the distributions of SADT loads. It is seen that the sets A003-36 km/A002-225 km, A011-90 kmA002-225 m, A004-22 km/A003-36 km, A009106 km/A003-36 km, A011-90 km/A003-36 km sets A010-84 km/A007-12 $\mathrm{km}$ and A004-90 $\mathrm{km} / \mathrm{A} 003-36 \mathrm{~km}$ show similarities. This can be clearly seen from the axle load distribution curves in Figures 5 and 6.

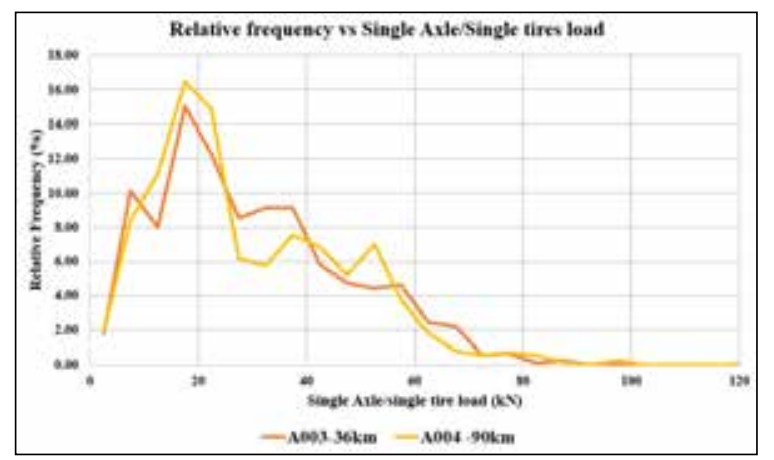

Figure 6 - SAST Axle Load Distribution at Locations A004-90 km and A003-36 km

and A011-90 km/A009-106 km show similarities in load distributions. These observations can be clearly seen from the axle load distributions shown in Figures 7 and 8.

Table 4 - Squared Euclidean Distance Matrix for SADT Load Distributions

\begin{tabular}{|c|c|c|c|c|c|c|c|c|c|c|c|c|c|c|c|}
\hline \multirow[b]{2}{*}{ Case } & \multicolumn{15}{|c|}{ Squared Euclidean Distance } \\
\hline & 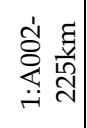 & 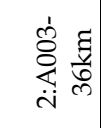 & 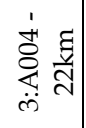 & 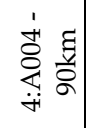 & 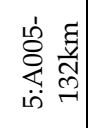 & 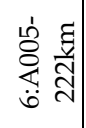 & 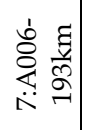 & 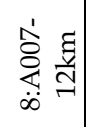 & 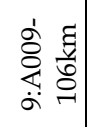 & 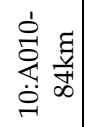 & 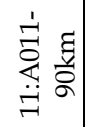 & 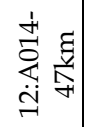 & 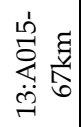 & 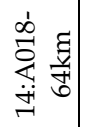 & 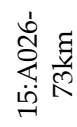 \\
\hline \multicolumn{16}{|l|}{ 1:A002-225km } \\
\hline 2:A003-36km & 0.005 & & & & & & & & & & & & & & \\
\hline 3:A004 -22km & 0.008 & 0.005 & & & & & & & & & & & & & \\
\hline 4:A004 -90km & 0.01 & 0.007 & 0.012 & & & & & & & & & & & & \\
\hline 5:A005-132km & 0.024 & 0.018 & 0.02 & 0.015 & & & & & & & & & & & \\
\hline 6:A005-222km & 0.011 & 0.011 & 0.008 & 0.02 & 0.036 & & & & & & & & & & \\
\hline 7:A006-193km & 0.009 & 0.008 & 0.012 & 0.007 & 0.019 & 0.014 & & & & & & & & & \\
\hline 8:A007-12km & 0.023 & 0.019 & 0.025 & 0.011 & 0.009 & 0.035 & 0.02 & & & & & & & & \\
\hline 9:A009-106km & 0.009 & 0.003 & 0.01 & 0.008 & 0.019 & 0.015 & 0.008 & 0.024 & & & & & & & \\
\hline 10:A010-84km & 0.02 & 0.016 & 0.02 & 0.014 & 0.016 & 0.033 & 0.021 & 0.006 & 0.026 & & & & & & \\
\hline 11:A011-90km & 0.005 & 0.005 & 0.011 & 0.009 & 0.019 & 0.012 & 0.008 & 0.02 & 0.005 & 0.024 & & & & & \\
\hline 12:A014-47km & 0.021 & 0.024 & 0.026 & 0.033 & 0.056 & 0.011 & 0.023 & 0.048 & 0.025 & 0.048 & 0.022 & & & & \\
\hline 13:A015-67km & 0.022 & 0.016 & 0.016 & 0.021 & 0.04 & 0.024 & 0.027 & 0.046 & 0.017 & 0.046 & 0.019 & 0.044 & & & \\
\hline 14:A018-64km & 0.016 & 0.012 & 0.017 & 0.012 & 0.019 & 0.025 & 0.008 & 0.023 & 0.012 & 0.023 & 0.014 & 0.031 & 0.031 & & \\
\hline 15:A026-73km & 0.02 & 0.023 & 0.028 & 0.018 & 0.023 & 0.025 & 0.017 & 0.016 & 0.025 & 0.024 & 0.018 & 0.026 & 0.046 & 0.016 & \\
\hline 16:A027-9km & 0.017 & 0.009 & 0.014 & 0.017 & 0.01 & 0.024 & 0.017 & 0.019 & 0.012 & 0.022 & 0.01 & 0.039 & 0.029 & 0.02 & 0.028 \\
\hline
\end{tabular}




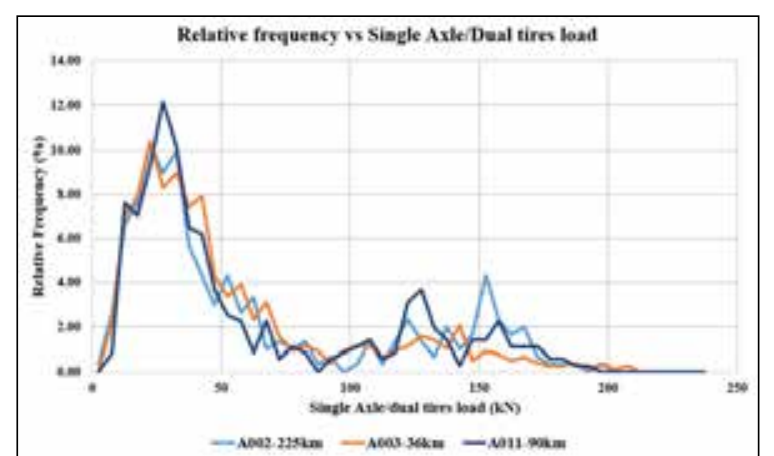

Figure 7 - SADT Axle Load Distributions at Locations A003-36 km, A002-225 km \& $\mathrm{A} 011-90 \mathrm{~km}$

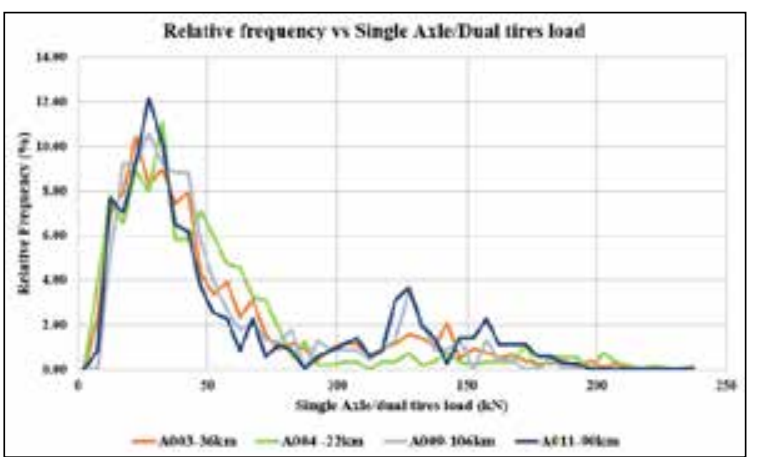

Figure 8 - SADT Axle Load Distributions at Locations A003-36 km, A004-22 km, A009-106 km \& A011-90 km

\section{Summary}

From the distributions of axle counts, it was found that in all axle load survey locations, on average $57 \%$ of the axles were single axles with single tires, $38 \%$ were single axles with dual tires, $4.9 \%$ were tandem axles with dual tires and only $0.1 \%$ constitutes tri axles with dual tires. This composition can be used to determine the traffic data input to M-E design when the manual classified counts are available.

It was seen from the cluster analysis that there are certain representative locations which has the same axle load distributions. Accordingly, the SAST axle load distributions in A010 and A007 roads were similar. Also, SAST axle load distributions in A004 and A003 roads were similar. The SADT axle load distributions in A003, A002 and A011 were having similar distribution patterns and also A003, A004, A009 and A011 were having similar axle load distributions. Through these observations it can be predicted that there could be regions in the island where there are similar axle load distributions.

However, as per the observations in this study, the similar axle load distributions were seen not in the locations in proximity. Therefore, further axle load survey data collection in the nearby areas is required and further analysed by clustering, in order to determine whether the axle load distributions of locations in a particular region show similarities. Using the methodology followed in this study, traffic loading zones could be identified if the axle load distributions in a particular region show similarities.

\section{References}

1. AASHTO, M., 2008. Mechanistic-Empirical Pavement Design Guide, A manual of Practice. Interim ed. s.l.:American Association of State Highway and Transportation Officials.

2. Mirza, W. M., Hafeez, I. \& Kamal, M. A., 2011. Comparative Study of Empirical and Mechanistic-Empirical Pavement Design Methodology Using Kenlayer Software. International Journal of Pavement Engineering and Asphalt Technology, 12(2), pp. 50-62.

3. Hall, K. D., Xiao, D. X., Pohl, E. A. \& Wang, K. C. P., 2012. Reliability-based mechanistic-empirical pavement design with statistical methods. Transport Res Rec: J Transport Res Board, 2305(1), pp. 121-130.

4. Carvalho, R. L. \& Schwartz, C. W., 2006. Comparisons of Flexible Pavement Designs. Journal of Transportation Research Board, 1947(1), pp. 167-174.

5. Kulkarni, R. B., 1994. Rational Approach in Applying Reliability Theory to Pavement Structural Design. Transportation Research Record, Volume 1449, p. 13-17.

6. Timm, D. H. \& Newcomb, D. E. G. T. V., 2000. Incorporation of Reliability into MechanisticEmpirical Pavement Design. Transportation Research Record, 1730(1), pp. 73-80.

7. Austroads, 2019. Guide to Pavement Technology Part 2: Pavement Structural Design. 4.3 ed. Sydney, NSW: National Association of Australian State Road Authorities.

8. Soetewey, A., 2020. Towards Data Science. [Online]

Available at:

https://towardsdatascience.com/the-completeguide-to-clustering-analysis-10fe13712787 [Accessed 2805 2020].

9. IBM, 2020. IBM SPSS Statistics 25. [Online] Available at: https://www.ibm.com/support/pages/downlo ading-ibm-spss-statistics-25 [Accessed 10 January 2021]. 